Известия НАН Армении, Физика, т.57, №1, с.52-58 (2022)

УДК 536.756

DOI:10.54503/0002-3035-2022-57.1-52

\title{
ТЕРМОДИНАМИКА ФИЗИЧЕСКИХ ПРИБЛИЖЕНИЙ НЕДЕТЕРМИНИСТИЧЕСКИХ ПОЛИНОМНЫХ ПОЛНЫХ ЗАДАЧ
}

\author{
В.А. СТЕПАНЯН ${ }^{*}$ С.Г. ХАЧАТРЯН, С.А. ОГАНИСЯН \\ Американский Университет Армении, Ереван, Армения \\ *e-mail: varostep@gmail.com \\ (Поступила в редакцию 15 июня 2021 г.)
}

\begin{abstract}
Недетерминистические полиномные полные задачи играют важную роль в современной коммуникации, безопасности и многих других областях. Одной из наиболее известных НП-полных задач является задача составления экзаменационного расписания. В задаче дана информация о предметах и о студентах, которые записались на некоторые из них, а также временные интервалы зарезервированные для экзаменов по этим предметам. Необходимо найти такое расписание, при котором ни у одного из студентов не будет одновременно зарезервировано несколько экзаменов. Мы моделируем эту НП-полную задачу как одномерную систему частиц. Добавив некоторые взаимодействия между частицами на основе данных задачи, мы решаем уравнения движения до достижения системой равновесия. Затем, мы используем состояние равновесия, чтобы собрать частицы вместе так, чтобы каждый кластер представлял собой один временной интервал. Чтобы рассмотреть физику этой модели, мы используем метод реплик, с целью найти функционал свободной энергии в этой системе. Формулируется гипотеза, что все или, по крайней мере, большинство численных методов возможно описать в рамках этой модели с помощью соответствующего выбора взаимодействия между частицами.
\end{abstract}

\section{1. Введение}

Связи между физикой и НП-полными задачами часто обсуждалось в научных работах, от задач, касающихся НП-полноты самоорганизации белка [1] до квантовых вычислений НП задач [2]. Тем не менее, обсуждение в основном сосредоточено на том, является ли вычислительное моделирование какого-либо физического явления НП-полным. Всегда существует обратная связь, и здесь мы предлагаем физическую модель для комбинаторных задач, в частности НПполных задач. Существует множество приближенных решений НП-полных проблем, от случайно угадывающих алгоритмов до методов имитации отжига [3]. Для создания систематического подхода к способам сравнения различных математических моделей НП-полных задач, мы предлагаем объединить их в одну общую модель. 
Так как все НП-полные задачи по определению могут быть сведены друг к другу в полиномном времени [4], чтобы описать эту модель, мы обсудим одну из стандартных НП-полных задач. Возьмём множество экзаменов, множество студентов, которые должны сдать некоторые экзамены и $k$ временных рамок, доступных для этих экзаменов. Задача заключается в том, чтобы найти расписание, в котором ни одному из студентов не придется сдавать несколько экзаменов одновременно. Задача о составлении экзаменационного расписания - известная комбинаторная задача, которая по сути является НП-полной [4]. Существуют различные формулировки этой задачи [5,6]. Мы рассматриваем задачу без дополнительных ограничений, где даны списки студентов для каждого из $N$ предметов, исходя из которых нужно составить расписание из соответствующих $N$ экзаменов в $S$ временных рамках так, чтобы не было столкновений. Столкновение двух экзаменов происходит, если они назначены на один и тот же временной интервал, и по крайней мере один студент числится в обоих классных журналах. Начиная с 1960x годов было предложено много способов решения [7-11].

\section{2. Составление экзаменационного расписания без дополнительных ограничений}

Составление непротиворечивого расписания предполагает сильное взаимодействие между экзаменами. Мы предлагаем простое правило взаимодействия если происходит столкновение, экзамен переносится на следующий временной интервал. Чтобы сформулировать вычислительную модель, представим экзамены как одномерную динамическую систему $N$ взаимодействующих элементов, где временные интервалы будут дискретными циклическими координатами $x \in\{0,1, \ldots, S-1\}$. Таким образом, каждое расписание определяется вектором $\mathbf{X} \in\{1,2, \ldots, S-1\}^{N}$. Состояние экзамена $\alpha$ в данном расписании описывается его временным интервалом $x_{\alpha}$ и бинарной функцией $b_{\alpha}(x)$, которая показывает наличие столкновения при нахождении этого экзамена в $x_{\alpha}$. Единичное изменение $x_{\alpha}$ осуществляется простым одномерным отображением:

$$
f_{\alpha}\left(x_{\alpha}\right)=\left(x_{\alpha}+b_{\alpha}\right) \bmod S .
$$

Перемещение экзамена на произвольный временной интервал осуществляется последовательными итерациями отображения (1), которые действуют только на экзамен $\alpha$ и не меняют координаты остальных экзаменов. Для произвольного количества итераций $m$ индивидуальные отображения (1), применяемые последовательно ко всем экзаменам, определяют кумулятивное эволюционное $N$-мерное отображение $E$ :

$$
E_{m}(\mathbf{X})=\prod_{\alpha=0}^{N-1} f_{\alpha}^{(m)}\left(x_{\alpha}\right),
$$

где знак произведения обозначает $m$-кратное применение отображения (1) к каждому экзамену $\alpha=0 \rightarrow N-1$. Многомерное кумулятивное отображение (2) можно также рассматривать как одномерное отображение воздействующее на общее количество столкновений $c(\mathbf{X}) \rightarrow c\left(E_{m}(\mathbf{X})\right)$, где $0 \leq c(\mathbf{X}) \leq c\left(\mathbf{X}_{0}\right)$ и $\mathbf{X}_{0}-$ 
начальный $N$-мерный вектор распределения экзаменов. Таким образом, итеративное применение отображения $E_{m}^{(d)}(\mathbf{X}) \equiv E(m, d)(c)$ описывает эволюцию системы взаимодействующих экзаменов с начальным условием $E(m, 0)\left(c\left(\mathbf{X}_{0}\right)\right)$.

Численные эксперименты с эталонными данными Торонто [7] создают два типа траекторий. Хаотические траектории наблюдаются в случае со простых $m$ и $S$. В противном случае производится периодические траектории с короткой переходной фазой. Возможные расписания построены моделями удовлетворяющие поставленным требованиям, когда хаотические траектории сходятся $c(\mathbf{X})=0$ фиксированной точкой. Таким образом, становится важно работать над общим подходом для надежной оценки параметров модели, которые максимизируют скорость сходимости для удовлетворяющих моделей или указывают на их неадекватность.

\section{3. Физическая модель}

Рассмотрим модель бусинок на кольце - простую модель одномерного движения частиц по кругу. В задаче по составлению расписания частицы представляют собой экзамены, а замкнутое кольцо-множество временных интервалов. В этой моделе бусинки подвержены парному взаимодействию. Если существует по крайней мере один студент сдающий оба данных экзамена, то взаимодействие между экзаменами в этой паре отталкивающее, в обратном же случае - притягивающее. В результате этих парных взаимодействий достигается состояние термодинамического равновесия, в котором система экзаменов распадается на кластеры, каждый из которых представляет собой определенный временной интервал. Рассмотрим пример с пятью экзаменами. Парами экзаменов с общими студентами являются $\{(1,3),(1,4),(1,5),(2,3),(2,5),(3,5),(4,3),(4,5)\}$ (см. Рис.1).

Очевидно, что достигнутое состояние равновесия не является точным решением, поскольку один из трех интервалов все еще содержит 'плохую' пару $(1,4)$. Но это хорошее приближение, так как с помощью одного дополнительного шага его можно свести к окончательному решению.

В данной работе сформулирована гипотеза, что многие известные методы
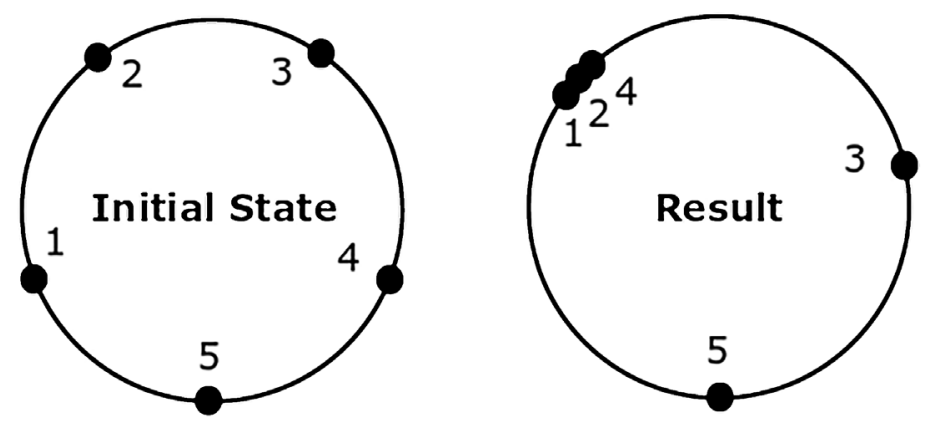

Рис.1. Задача расписания экзаменов 
математического моделирования для приближенных решений НП-полных задач могут быть сведены к модели бусинок на кольце с соответствующим выбором потенциала парных взаимодействий $\Delta\left\{x_{i}, x_{j}\right\}$. В частности, при аппроксимации колоколообразных потенциалов дельта функциями, цель симуляции сводится к нахождению ‘плохих' пар и изменению состояния системы к ближнее - соседнему состоянию, что соответствует методу градиентного спуска.

\section{1. Свободная энергия}

Чтобы изучить термодинамическое равновесие физической модели, рассмотрим задачу $N$ экзаменов со случайными данными. Гамильтониан этой модели запишется как

$$
-\beta \mathrm{H}=-\beta \sum_{i<j}^{1, N} \omega_{i j} \Delta\left\{x_{i}, x_{j}\right\},
$$

где $\beta=(K T)^{-1}, \Delta\left\{x_{i}, x_{j}\right\} \equiv \Delta_{i j}$ является потенциальной энергией между $i$-той и $j$ той бусинками, и зависит только от координат этих бусин, а $\omega_{i j}$ является коэффициентом взаимодействия между $i$-той и $j$-той бусинками, которое зависит от данных студентов и экзаменов, а значит является случайным. При определенном выборе потенциальной энергии реализуется Гамильтониан спиновых стекол.

Есть много подходов к решению таких систем [12,13]. В данной работе мы следуем методам стат. физики. Таким образом можем найти общие характеристики состояний равновесия конкретного типа конфигураций. Для такого обобщенного результата надо изучать систему со случайными коэффициентами. Мы будем считать распределение коэффициентов взаимодействия Гаусовым -

$$
\rho\left(\omega_{i j}\right)=\exp \left[-\frac{\left(\omega_{i j}-\bar{\omega}\right)^{2}}{2 \omega^{2}}\right] / \sqrt{2 \pi \omega^{2}}, \quad \mathrm{P}=\prod \rho,
$$

где пара $\omega, \bar{\omega}$ описывает процент пар экзаменов с общими студентами. В пределе $N \rightarrow \infty$, свободная энергия неупорядоченной системы подчиняется принципу самоусреднения [13]:

$$
-\beta F=\langle\ln Z\rangle_{\mathrm{P}}
$$

\section{2. Метод реплик, результаты и обсуждение}

Замороженную свободную энергию (5) можно вычислить, используя метод реплик [13]

$$
-\beta F=\lim _{n \rightarrow 0} \frac{\left\langle Z^{n}\right\rangle_{\mathrm{P}}-1}{n} .
$$

Следуя методу, описанному в [14], вычисляем $n$ репличную статсумму

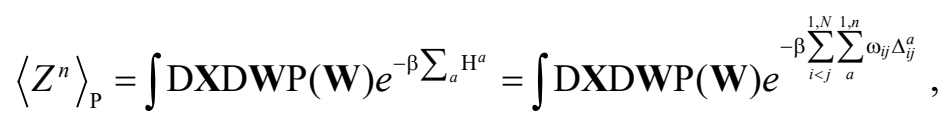

где 


$$
\begin{gathered}
\mathrm{DXDW}=d x_{1}^{1} d x_{2}^{1} d x_{3}^{1} \ldots d x_{N}^{n} d \omega_{1,1} d \omega_{1,2} d \omega_{1,3} \ldots d \omega_{N-1, N}, \\
\langle Z\rangle_{\mathrm{P}}^{n}=\exp \left[-\frac{\bar{\omega} N(N-1)}{4 \omega^{2}}\right] \int \mathrm{D} \mathbf{X} e^{\Lambda}, \\
\Lambda=\sum_{i, j}^{1, N}\left(\frac{\sum_{a} \beta \omega \Delta_{i j}^{a}-\frac{\bar{\omega}}{\omega}}{2}\right)^{2} .
\end{gathered}
$$

После этих обозначений мы получаем

$$
\begin{aligned}
& \Lambda=\left(\frac{\beta \omega}{2}\right)^{2} \sum_{a, b}^{1, n} \hat{\mathrm{Q}}_{a b}-\left(\frac{\beta \bar{\omega}}{2}\right) \sum_{a}^{1, n} \hat{\mathrm{M}}_{a}+\frac{\bar{\omega}^{2} N(N-1)}{4 \omega^{2}}, \\
& \hat{\mathrm{Q}}_{a b}=\sum_{i, j}^{1, N} \Delta_{i j}^{a} \Delta_{i j}^{b}, \quad \hat{\mathrm{M}}_{a}=\sum_{i, j}^{1, N} \Delta_{i j}^{a} .
\end{aligned}
$$

Пользуясь $\int \prod d \mathrm{M}_{a} \delta\left(\mathrm{M}_{a}-\hat{\mathrm{M}}_{a}\right) * \prod d \mathrm{Q}_{a b} \delta\left(\mathrm{Q}_{a}-\hat{\mathrm{Q}}_{a}\right)=1$, окончательно получаем

$$
\langle Z\rangle_{\mathrm{P}}^{n}=\int \mathrm{DQDM} \exp \left[\left(\frac{\beta \omega}{2}\right)^{2} \sum_{a, b}^{1, n} \mathrm{Q}_{a b}-\left(\frac{\beta \bar{\omega}}{2}\right) \sum_{a}^{1, n} \mathrm{M}_{a}+S\right],
$$

где $S=\ln \int \mathrm{DX} \delta\left(\mathrm{M}_{a}-\hat{\mathrm{M}}_{a}\right) \delta\left(\mathrm{Q}_{a}-\hat{\mathrm{Q}}_{a}\right)$.

Полученное уравнение (10) практически сводится к результату задачи самоорганизация белка, где свободная энергия модели бусинки на пружинках вычисляется с использованием метода реплик $[15,16]$, если принять $\Delta_{i j}=\delta\left(x_{i}-x_{j}\right)$. Разница заключается всего в двух аспектах. Во-первых, в рассмотренной модели отсутствуют пружины. Во-вторых, конечность координат достигается пространственной цикличностью, а не добавлением потенциальной энергии к Гамильтониану. Следуя результатам [15] для приближении среднего поля, дополнительная энтропия вычисляется как

$$
S=\ln \int \mathrm{D} \mathbf{X} \delta(\hat{\psi}-\psi)
$$

где

$$
\hat{\psi}\{\xi\}=\sum_{i=1}^{N} \prod_{a=1}^{n} \delta\left(x_{i}^{a}-\xi^{a}\right) .
$$

Так как в нашей задаче $x$ координаты принимают, по сути, ограниченные дискретные значения, мы можем интерпретировать функции как конечные последовательности. Следовательно, энтропия равна логарифму количества конфигураций координат, где $\hat{\psi}_{i} \equiv \hat{m}_{i}=m_{i} \equiv \psi_{i}$ для любого значения $i \in\left[1, t^{n}\right]$, где $t$ количество возможных значений координаты (количество временных интервалов в задаче по составлению расписания)

$$
\hat{\psi}\{\xi\}=\sum_{i=1}^{t^{n}} m_{i} \delta\left(\xi_{i}-\xi\right) .
$$


Количество конфигураций координат, где $\hat{\psi}=\psi$ эквивалентно количеству конфигураций, для которых последовательности $\left\{\hat{m}_{i}\right\},\left\{m_{i}\right\}$ являются одинаковыми. Важно отметить, что $\sum_{t=1}^{t^{n}} m_{i}=N$. Эту задачу можно свести к задаче, представленной ниже. Допустим, у нас есть $t^{n}$ коробок, в которые нужно разместить $N$ мячей, причем каждая коробка может вместить любое количество мячей. Нужно найти количество комбинаций для данной последовательности $\left\{m_{i}\right\}$, где $m_{i}$ количество мячей в $i$-той коробке. Решением этой задачи является

$$
\Omega=N ! / \prod_{i=1}^{t^{n}} m_{i} !
$$

из чего следует

$$
\begin{aligned}
& S=\ln \Omega=\ln N !-\sum_{i=1}^{t^{n}} \ln m_{i} ! \approx N \ln N+1-N-\sum_{i=1}^{t^{n}}\left(m_{i} \ln m_{i}+1-m_{i}\right)= \\
& =N \ln N+1-t^{n}-\sum_{i=1}^{t^{n}} m_{i} \ln m_{i} .
\end{aligned}
$$

В общем случае, для любого типа $\Delta_{i j}$ можно сказать, что термодинамическое равновесие для данных $\omega$ и $\bar{\omega}$ совпадает с той матрицей $\Delta_{i j}$, которая максимизирует

$$
\int \mathrm{D} Q D M \exp (-\beta F)
$$

где

$$
-\beta F=\frac{\beta^{2} \omega^{2}}{4} \sum_{a, b}^{1, n} \mathrm{Q}_{a b}-\frac{\beta \bar{\omega}}{2} \sum_{a}^{1, n} \mathrm{M}_{a}+S .
$$

Сходимость метода или скорость самоорганизации нашей модели напрямую зависит от выбора потенциалов $\Delta\left\{x_{i}, x_{j}\right\}$.

\section{4. Заключение}

Главной мотивацией к данной работе явилась задача по составлению экзаменационного расписания. Мы рассматриваем НП-полные задачи как системы взаимодействующих частиц, движущиеся в одном измерении. Взаимодействия выбираются на основе ограничений задачи таким образом, чтобы система релаксировала в сторону состояния равновесия, которое рассматривается как приемлемое решение. Свободная энергия модели, в зависимости от выбора потенциала, вычисляется с использованием метода реплик.

Предлагаемая модель может быть сведена к различным итеративным алгоритмам соответствующим выбором конкретных потенциалов взаимодействия. Рассмотрим, например, метод симулированного отжига или любой другой метод приближения похожий на градиентный спуск с данной функцией $f$. Взяв дельтаобразные потенциалы, получим довольно точное представление этих методов. Явный вид формы функции потенциала будет завесить от выбора функции $f$. Если взять метод градиентного спуска, где $f$ обозначает количество «плохих» пар в системе, то этот метод можно будет свести к модели с потенциалом $\Delta\left\{x_{i}, x_{j}\right\}=\delta\left(x_{i}-x_{j}\right)$. 
Гипотеза о том, что любой метод можно свести к этой модели, будет предметом обсуждения следующей работы.

\section{ЛИТЕРАТУРА}

1. J. Bahi, W. Bienia, N. Cote, C. Guyeux. J. Bioinform. Comput. Biol. 12, 135 (2014).

2. S. Aaronson. SIGACT News, 36, 30 (2005).

3. J.M. Thompson, K.A. Dowsland. Ann. Oper. Res., 63, 105 (1996).

4. M.R. Garey, D.S. Johnson. Computers and Intractability; A Guide to the Theory of NPCompleteness, W.H. Freeman \& Co., 1990.

5. A. Schaerf. Artif. Intell. Rev., 13, 87 (1999).

6. B. McCollum. Proceedings of the 6th International Conference on Practice and Theory of Automated Timetabling VI. PATAT'06, Springer-Verlag, Berlin, Heidelberg, p. 373, 2006.

7. R. Qu, E. Burke, B. McCollum, L. Merlot, S. Lee. J. Schedul., 12, 55 (2009).

8. M.W. Carter, G. Laporte, S.Y. Lee. J. Oper. Res. Soc., 47, 373 (1996).

9. L.T.G. Merlot, N. Boland, B.D. Hughes, P.J. Stuckey. Practice and Theory of Automated Timetabling: Selected Papers from the 4th International Conference, Springer Lecture Notes in Computer Science, p. 2740, 2003.

10. Z. Ulker, E. Ozcan, E. Korkmaz. Practice and Theory of Automated Timetabling. Selected Papers from the 6th International Conference, Springer Lecture Notes in Computer Science, p. 3867, 2006.

11. K. Dowsland, J. Thompson. J. Oper. Res. Soc., 56, 426 (2005).

12. A.S. Gevorkyan, V.V. Sahakyan. J. Contemp. Phys., 55, 265 (2020).

13. K. Binder, A.P. Young. Rev. Mod. Phys., 58, 801 (1986).

14. M. Mezard, G. Parisi, M. Virasoro. Spin Glass Theory and Beyond, World Scientific, 1986.

15. A.M. Gutin, A.Y. Grosberg, E.I. Shakhnovich. J. Phys. A: Math. Gen., 26, 1037 (1993).

16. Y.Sh. Mamasakhlisov, S. Bellucci, S. Hayryan, H. Caturyan, Z. Grigoryan, C.-K. Hu, Eur. Phys. J. E., 38, 100 (2015).

\section{THERMODYNAMICS OF PHYSICAL APPROXIMATIONS TO NON DETERMINISTIC POLYNOMIAL COMPLETE PROBLEMS}

\section{V.A. STEPANYAN, S.G. KHACHATRYAN, S.A. HOVHANNISYAN}

Non Deterministic Polynomial Complete problems are playing an essential role in nowadays communications, security and many other fields. One of the well-known NPcomplete problems is the Examination Timetabling problem. Given the information about the courses and the students that have taken some of those courses as well as the time slots for the exams of those courses the problem asks if there is a timetable such that no student has two exams simultaneously. We are modeling this NP-complete problem as a system of particles with one dimensional motion. After adding some interactions between the particles based on the problem we are simulating the motion and arriving to an equilibrium. We then use the equilibrium state to group the particles together where each cluster represents a time slot. To discuss the physics of this model we use the Replica method to find the free energy functional of this system. A hypothesis is formulated that all or at least most numerical solutions for NPcomplete problems can be brought to this model with some configuration of interactions between the particles. 